\title{
Is medical treatment of Alcohol Withdrawal Syndrome a Stag Hunt? Challenges and opportunities in managing risk and uncertainty in addiction cessation
}

This article was published in the following Dove Press journal:

Risk Management and Healthcare Policy

\section{Roger Lee Mendoza}

School of Business, Wilmington University, New Castle, DE, USA
Correspondence: Roger Lee Mendoza School of Business, Wilmington University, 3 I Reads Way, New Castle, DE 19720, USA

Email roger.I.mendoza@wilmu.edu
Purpose: While the individual and social costs of alcoholism or alcohol use disorder are well established, few are aware that medical problems can arise during detoxification, some of which can be life-threatening. This study determines if sustained treatment for Alcohol Withdrawal Syndrome (AWS) might be based on the strategic choices and expectations of patients and health care providers alike, as well as the risk mitigation options available to them.

Design/approach: AWS was modeled as a Stag Hunt to explain both risk and decision-making in medical treatments for detoxification, since it can deduce a set of equilibrium strategies available to both patient and provider. Modeling was based on a review of juried literature gathered from search engines with the use medical subject heading terms.

Main findings: While there is little evidence that decision-making is shared between patient and physician in AWS treatments, the outcomes of their interactions depend on utility-maximizing choices each makes in anticipation of the other. Payoff-dominant and risk-dominant treatment outcomes are equally likely and equally cost-efficient, as conditioned by the presence (or absence) of mutual trust and assurance in reciprocal transactions.

Conclusion/value: Simulation games, such as the Stag Hunt, offer a viable framework to understand patient and provider incentives and health-affecting behaviors during treatments for addiction cessation. If both anticipate indefinitely interacting in the absence of any predetermined or foreseeable final visit, they can maximize future payoffs from mutual cooperation and accountability, which fosters health promotion. However, this study suggests that the effect of cooperation is distinct from the effect of time in AWS and other addiction-cessation programs. Keywords: health-affecting behaviors, healthcare provider, patient compliance, payoff, relapse, simulation game, treatment disruption/discontinuation

\section{Introduction}

By the definition of the World Health Organization, alcoholism or alcohol use disorder refers to any form of alcohol consumption that results in health and other problems. Whether classified as mild, moderate, or severe, alcohol consumption becomes risky when "warning signs" appear. These include craving for alcohol when not drinking, putting it above personal responsibilities, spending a substantial amount of resources (money, time, effort) in consuming it, behaving differently after drinking, and experiencing temporary blackouts or short-term memory loss from drinking. ${ }^{1}$

Alcoholism is one of the most common forms of substance abuse and dependence worldwide. In many countries, it is a pervasive, age-old problem. ${ }^{2}$ In the USA alone, over 
$27 \%$ of the population aged 12 and older are considered risky drinkers. ${ }^{3}$ Alcoholism could also be an unconscious attempt at self-treatment for other issues such as depression. ${ }^{4}$ The magnitude of the health, sociopsychological, and economic costs of alcoholism extends far beyond the chronic drinker. Car crashes, domestic violence, as well heinous crimes have often been linked to alcohol use disorder. ${ }^{5,6}$ Its individual costs and externalities have led to government intervention, particularly in the form of policies that influence availability and price of alcoholic beverages, drinking context, and perceived risks of heavy drinking. ${ }^{7}$ Yet, the most recent OECD global health report finds that "there has been little success in tackling [...] harmful alcohol use" and its associated consequences. ${ }^{8}$

Wthdrawal from chronic drinking can also lead to major health problems. A characteristic withdrawal syndrome ensues with typical clinical features when an alcohol-dependent individual abruptly terminates or substantially reduces his/her alcohol consumption. ${ }^{9,10}$ It is estimated that about half of alcoholics seeking abstinence will develop withdrawal symptoms. ${ }^{11}$ While the efficacy of treatment approaches to alcohol use disorder has been widely studied, along with sensitization and educational interventions to stop them, ${ }^{12}$ less attention has been paid by the medical or scientific community on why treatment for alcohol withdrawal effects might be unsuccessful. ${ }^{13}$ Effective therapy for alcoholism, for example, is attributed to a wide range of factors. These include the individual's readiness to commit to treatment (i.e., ability to realize the addiction problem and take steps toward change), self-efficacy (i.e., the trust or confidence that difficult situations and challenges will be overcome), expectation level (i.e., expectation of the treatment results and satisfaction gained from these results during and at the end of the therapeutic procedure/s), social support, and clinical profile. ${ }^{14}$ Yet, common threads among these factors, such as the role and influence of emotions, remain unexplored. ${ }^{14}$ In addition, why and how these factors might affect the choices to pursue or discontinue treatment for alcohol withdrawal on the part of the patient and physician need to be addressed.

This study inquires into Alcohol Withdrawal Syndrome (AWS) - also known as discontinuation syndrome - from a simulation game approach. The importance of simulation game to any addiction cessation stems from providing a powerful conceptual framework for modeling control behavior, including specifying the strategies to be played and the conditions to be satisfied for particular traits to evolve. ${ }^{15}$ The simulation game approach is, therefore, transactional, rather than purely clinical, particularly in examining the emotional basis of patient-provider relationships. It can successfully describe and explain the costs and benefits of varying strategies and the dynamics for establishing equilibria in a number of patient-provider interactive scenarios. It serves as a useful tool in health care risk management.

The problem for analytical investigation in this study is whether, and why, treatment of AWS might be based on the strategic and interactive choices and expectations of alcoholics and health care providers alike, as well as the risk mitigation options available to them. It offers theoretical and practical insights about value and behavior in the production and consumption of health and health care relative to chronic drinkers seeking abstinence, but encountering difficulties along the way. In furthering our understanding of health care systems and health-affecting behaviors, the implications of this study extend to many other addiction-cessation programs and treatments, besides alcoholism.

\section{Treatment of alcohol withdrawal}

AWS occurs among alcoholics who abruptly stop or significantly reduce alcohol consumption. When chronic alcohol consumption is suddenly stopped or reduced, the brain's neurotransmitters (i.e., message-transmitting brain chemicals) previously suppressed by alcohol are no longer suppressed. They rebound, resulting in brain hyperexcitability. Among others, anxiety, irritability, agitation, tremors, and seizures result directly from brain hyperexcitability. As stress hormones are overproduced, the sudden removal of the central nervous system depressant could potentially be life-threatening., ${ }^{2,9}$ Withdrawal delirium, also known as delirium tremens (DTs), is considered the most severe and dangerous symptom. The death rate from DTs (which initially causes confusion, rapid heartbeat, excessive sweating, and fever) ranges from $1 \%$ to $5 \%{ }^{16}$

Alcohol detoxification often takes from 7 to 10 days at the minimum. ${ }^{3}$ Manifestations of AWS arise at the onset of detoxification, which can be broken down into three stages, each with its associated symptoms, as presented in Figure 1. These symptoms are obviously the reverse of those associated with alcohol consumption.

Major complications could arise in Stage 3, especially if the individual has existing health conditions such as infections, heart disease, lung disease, or a history of seizures. Withdrawal symptoms also tend to be more severe among alcoholics who have undergone prior multiple episodes of AWS, a process known as the "kindling effect".,10 Because any AWS symptom can rapidly worsen, medical treatment is critical even if the symptoms are seemingly mild. The severity of AWS symptoms depends on how much and for how long an individual has been chronically drinking. ${ }^{17}$

Medical treatment for individuals with AWS starts with a complete medical history review (including substance 


\begin{tabular}{|c|c|c|}
\hline Stage & Typical signs/symptoms & Duration \\
\hline 1 Mild & $\begin{array}{l}\text { Anxiety, insomnia, nausea, } \\
\text { mood swings, loss of } \\
\text { appetite, depression, } \\
\text { abdominal pain }\end{array}$ & $\begin{array}{l}8 \text { hours after last } \\
\text { alcoholic drink }\end{array}$ \\
\hline 2 Moderate & $\begin{array}{l}\text { High blood pressure, } \\
\text { increased body temperature, } \\
\text { excessive sweating, unusual } \\
\text { heart rate, irritability, confusion }\end{array}$ & $\begin{array}{l}24-72 \text { hours after } \\
\text { last alcoholic drink }\end{array}$ \\
\hline 3 Severe/delirium tremens & $\begin{array}{l}\text { Various types of } \\
\text { hallucinations, fever, } \\
\text { seizures, severe agitation, } \\
\text { major depression }\end{array}$ & $\begin{array}{l}>72 \text { hours after last } \\
\text { alcoholic drink. } \\
\text { May persist for } \\
\text { weeks (sometimes } \\
\text { months) if left } \\
\text { untreated }\end{array}$ \\
\hline
\end{tabular}

Figure I Progression stages of Alcohol Withdrawal Syndrome.

abuse history and any mental health conditions) and physical examination (usually including complete blood work and urine screening). They help the attending physician or health care provider team determine if the patient is suffering from AWS and, if so, its severity. Assessment tests, such as the so-called Clinical Institute Withdrawal Assessment (CIWA) scale, are typically employed to determine treatment and whether symptoms might progress in severity. Pharmacological treatment is equally important. Benzodiazepines, the drugs of choice in many AWS treatments, can prevent seizures and DTs. ${ }^{18,19}$

Mild to moderate withdrawal symptoms are often treated by a doctor in an outpatient setting, especially if complications are absent and the patient has supportive family and friends. Inpatient detoxification for high-risk patients is often performed at a hospital or other facility (e.g., psychiatric facility). In this case, the physician frequently works with a health care team that includes nurses and mental health specialists. Whether outpatient or inpatient, the major objectives of medical treatment of AWS are to: 1) reduce immediate withdrawal symptoms; 2) prevent complications; and 3) initiate long-term therapy to promote total alcohol abstinence..$^{20}$

Time is key to treatment adherence, whether it is based on a certain number of treatment sessions, ${ }^{21}$ treatment with a specific number of days, ${ }^{22}$ or a continuous measure related to time spent in treatment. ${ }^{23}$ Studies indicate that the majority of AWS patients gain significant improvement only at the third month of or following treatment. ${ }^{24}$ The type and frequency of monitoring is guided by symptom severity as well as the characteristics of the patient and his/her environment. ${ }^{9,10}$ Most patients require daily evaluation (whether by their doctor and other providers or by means of structured self-evaluation) until their symptoms decrease and the medication dosage is reduced. Patients with advanced Stage 2 or Stage 3 alcohol withdrawal need closer monitoring, some in an intensive care setting. Their vital signs, heart rhythm and rate, respiration, fluid and electrolyte balance, blood glucose level, skin, elimination, mental and neurological status, and nutritional status have to be closely monitored. ${ }^{25}$

Noncooperation or noncompliance on the part of the patient could result in periodic treatment disruptions and premature termination. Studies find that it is not unusual for drop-out rates to exceed $50 \%$, especially during the first month of any form of treatment. ${ }^{24,26}$ However, treatment drop-out rates can widely vary based on systemic, sociocultural, and structural obstacles that a patient might face. For instance, they account for a higher drop-out rate (31\%) among women. This is because women typically assume more domestic, especially child care, responsibilities, tend to have less health care coverage, face greater stigma and social prejudice for alcohol use disorder and detoxification, seek more nonspecialized health services, and have higher rates of mental disorder associated with the use of psychoactive substances that might make treatment more challenging. ${ }^{24,27,28}$ 
In terms of race, black women appear to be at higher risk of dropping out of therapy for economic reasons besides those previously cited. ${ }^{27,29}$

Health care providers can also be impeded from further treating a patient with AWS. Physicians and other providers might have limited qualifications or experience in treating detox patients ${ }^{30}$ or are simply reluctant to diagnose a disorder such as AWS that they are not confident they can treat or could breed potential malpractice suits and complaints. ${ }^{31}$ Equally important factors that could impede treatment are physician location, cost, and waiting list, ${ }^{32,33}$ as well as the lack of integration between primary care and mental health programs. $^{34}$

A transactional framework that comprehends both patient and provider incentives and behaviors is critical in managing risks and uncertainty associated with AWS treatment, particularly disruptions and terminations. Such a model should offer valuable practical and theoretical insights to health care practitioners, patients, as well as researchers of AWS. Nonetheless, AWS treatment constitutes an initial step toward long-term abstinence. It does not resolve per se the underlying problem of alcohol abuse or dependence. On the contrary, studies suggest that abstinence is unlikely if the patient does not enroll in full treatment and rehabilitation for alcoholism, which may include individual counseling and therapy, group sessions (e.g., Alcoholic Anonymous), and long-term medications to reduce the risk of relapse. ${ }^{2,35}$

\section{The risk management model}

We propose for this study a game-simulation of cooperation in the medical treatment of AWS. Game-theoretic modeling helps predict whether such treatment (outpatient or inpatient) is likely to be costly or inefficient to the patient and provider. It is particularly useful for repetitive or rhythmic behaviors that occur over a period of time, ${ }^{15}$ as exemplified by AWS treatments. This is because it helps explain disrupted or prematurely terminated AWS treatments that lead to patient drop-out, especially in terms of value and corresponding behaviors in the production and consumption of health and health care.

Pioneered by mathematicians Emile Borel and John Von Neumann in the 1920s, game theory gradually evolved to discover whether a "best" or "optimal" strategy exists for any problem of social cooperation (the "game") and to find that optimum. It was initially intended for economists, but its applications to politics, psychology, sociology, warfare, recreational games, and other fields, such as evolutionary biology, soon became apparent. Games and strategy matrices were identified and developed by its theorists over time, based mainly on "cooperation" among players (including compliance with rules, norms, and expectations) or mutual "defection" (as a result of conflict and competition). ${ }^{36}$

Any simulation game model contains five basic elements: 1) competitive "players" (or decision-makers); 2) "rules" governing players' behaviors; 3 ) "strategies" available to each player within the given set of rules; 4) game "outcomes" (each of which is a result of particular interactive choices made by players at a given point in the game); and 5) "payoffs" accrued by each player as a result of each possible outcome. Along with a solution concept, these elements allow game theorists to deduce a set of equilibrium strategies for each player. Thus, when any game strategy is employed, a so-called "Nash Equilibrium" (or "Equilibria") will arise where no single player or set of players may further gain by unilaterally deviating from that strategy.

We propose the Stag Hunt as a useful analytical framework in managing risks associated with substance abuse rehabilitation. The Stag Hunt models conflict between the goals of safety and cooperation by examining trade-offs that arise where a larger - but demonstrably costlier or riskier payoff can be generated from one strategy (e.g., sustained AWS treatment) in contrast to another (alcohol relapse). With two players (or sets of players) and two choices of actions and outcome-based preferences, Stag Hunt modeling for AWS allows us to identify a dominant solution/s for a predefined setting based on the essential prerequisites of simulation games: 1) players have a common interest to score as high as possible (i.e., maximize their respective gains/benefits); 2) however, they also have competing interests to increase their respective proportion of scores; 3 ) each player's utilitymaximizing strategy requires consideration of the other players' choices; and 4) strategy choice is always made under conditions of risk and uncertainty. ${ }^{37}$

In the classic Stag Hunt, two hunters are faced with the dilemma of bagging either a stag or a hare. If one hunter chooses a stag, s/he would require the cooperation of the other in order to succeed. Going for a hare, on the other hand, can be a unilateral decision, but the prize is considerably less than the stag. Having tracked a stag and discovered its path in the forest, the hunters can mutually cooperate in capturing the stag, which offers them a big, tastier meal that will last them for days to come. However, a long day passes without a trace of the stag, although the hunters are reasonably certain that it will eventually come. In the meantime, the hunters see a hare (or two) running back and forth on the same path where they have laid out the stag trap. While a hare is an easy catch, and 
the temptation to go for it is high after a long, tiring day of waiting for the stag, it will result in the stag trap being wasted for a small meal that can only feed (rather insufficiently) one of the hunters. Obviously, too, the longer the stag wait, the greater is the temptation for either one or both hunters to settle for a hare. In fine, the risk-utility trade-off is: risk the stag never coming, or risk another hunter taking the kill. ${ }^{38}$

A Nash Equilibrium is said to exist where a player's strategy choice is a best response compared to other available choices, so that no player has anything else to gain by changing his/her choice unilaterally. ${ }^{38}$ Two pure strategy Nash Equilibria exist in the Stag Hunt. We have denoted each Nash Equilibrium with an asterisk in Figure 2.

Assume that the meal payoff from capturing a stag is 25 times greater than a hare (payoff=2). If both hunters cooperate and go for the stag despite the long, uncertain wait ( $a, w>b, c, d, x, y, z)$, each of them maximize their payoffs $(50,50)$. If one hunter acts unilaterally by catching the hare, $\mathrm{s} /$ he gains at the expense of the other who continues to wait for the stag $(2,0 ; 0,2)$. When the hunters mutually "defect", and instead go for the hare/s, each one still gains $(d, z>b, y)$. However, the individual payoff is quite small compared to a stag. It becomes even smaller if there is just one hare to catch, which they will have to agree to divide between themselves $(1,1)$. Given the essential prerequisites of a Stag Hunt, the Nash Equilibrium shown in Figure 2 can either be payoff-dominant $(a, w)$ or risk-dominant $(d, z)$, as each hunter anticipates which strategic option the other will choose and how to maximize his/her expected payoff in light of the attendant risks and uncertainties.

Through Stag Hunt modeling, we next examine whether the strategic solution is one of payoff-dominant cooperation or risk-dominant defection when a doctor or other providers offer, and a patient receives, medical treatment for AWS and why.

\section{Treatment issues and obstacles}

We find that medical treatment of AWS offers one illustrative - albeit counterintuitive - case of a Stag Hunt after reviewing the relevant literature. We searched PubMed for juried studies published in English covering AWS treatment and management in humans without any restriction on the date of publication. Of specific interest in this literature search were treatment disruptions and premature terminations (i.e., why and how?). We relied on the following medical subject heading terms as representative variants of AWS: "alcoholism", "alcohol withdrawal seizures" and "alcohol withdrawal delirium" and "drug therapy." Peer-reviewed articles that were deemed irrelevant were excluded based on their titles and abstracts. Full-text articles were subsequently gathered for the 17 articles considered relevant to clinical treatment and management. Lastly, cross-references indicated in these full-text articles were checked for other pertinent studies. Literature on the subject matter remains scant to date.

From the literature review, two key variables, trust and assurance - on a mutually reciprocal basis - appear to be crucial in breeding and sustaining cooperation during AWS treatment. They encourage and sustain cooperation between patient and provider, which generally drives continuous and successful AWS treatments. After all, "[h] ealthcare is, at its core, based on relationships. And, as with any relationship, trust is foundational to building and maintaining a strong relationship. The very personal nature of healthcare relationships supports the importance of trust". ${ }^{39}$ Trust (which encompasses confidence, loyalty, regard, and empathy) can nonetheless be fragile. While trust is a crucial factor that characterizes an ongoing depth of relationship between patient and provider, assurance is a related factor that develops or maintains such relationship. Within its scope lie integrity, longitudinal care, patients' consultation experiences, and mutual satisfaction. ${ }^{40}$

\begin{tabular}{|c|c|c|}
\hline Players & \multicolumn{2}{|c|}{ Hunter 2 (or patient) } \\
\hline Hunter 1 (or doctor) & Stag & Hare \\
\hline Stag & $a, w(50,50)^{\star}$ & $b, x(0,2)^{\star}$ \\
\hline Hare & $c, y(2,0)^{\star}$ & $d, z(1,1)^{\star}$ \\
\hline
\end{tabular}

Hence, $a>c \geq d>b$; $w>x \geq z>y$

${ }^{*}$ Nash Equilibrium

Figure 2 Payoff matrix in classic Stag Hunt. 
For instance, studies report that lack of empathy and confidence in the provider/s as well as doubts on treatment efficacy (diminished assurance) significantly increase the likelihood of patient drop-out. We have cited literature in the "Treatment of alcohol withdrawal" section to indicate how providers might be impeded from seeing the patient on an ongoing or long-term basis as a result of limited qualifications, experience, location, cost, wait list, and unintegrated primary care and mental health strategies and programs. ${ }^{30-34}$

Conversely, patients who have a strong relationship or form a bond with their providers are more likely to continue their prescribed treatment. ${ }^{24}$ However, another study shows that despite physicians' attempts to form an empathic bond with their patients, several obstacles can occur: $39.1 \%$ of the physicians did not explain the problem in a clear and attentive manner; $58 \%$ of the physicians did not check the patients' level of understanding about the diagnostic information; $53 \%$ of the physicians did not ask the patient about therapeutic indications; and $50 \%$ of the physicians did not take into account psychiatric and psychosocial problems. ${ }^{34}$ They lead to reduced patient trust and assurance and tend to raise patient compliance issues.

From a clinical perspective, outpatient treatment of mild (Stage 1) to moderate (Stage 2) AWS is generally considered safe and less costly than inpatient treatment for those who are at high risk. Successful treatment requires the outpatient's commitment to taking prescribed oral medications and frequent follow-up visits and consultations with the doctor. Family support, especially in terms of administering medication and monitoring patient compliance with the doctor's advice, is also very important. ${ }^{35}$ AWS signs and symptoms can easily cause fear and anxiety in both patients and family members. However, family dysfunction (e.g., domestic problems) or home triggers for alcohol relapse diminish the likelihood of completed treatment, as family support becomes scarce and/ or reinforcing behavior surfaces. These often lead to missed appointments and noncompliance with prescribed medication and physician advice. ${ }^{41}$ Continuous evaluation by a mental health provider or team is critical in these instances. ${ }^{41}$

A study on outpatients in Denmark compared symptomtriggered versus fixed-schedule dosing for oral AWS medications. ${ }^{42}$ Researchers initially used chlordiazepoxide according to a fixed-dosage schedule, tapering the dose to zero after 8-10 days for each patient. No monitoring or systematic documentation of symptoms was undertaken, unless the patients complained of continuing clinical symptoms, which then led to dose alteration. The study discovered that a fixed-dosage scheme "suffers from the lack of individualized treatment, lack of monitoring and documentation of symptoms, and a paternalistic view hampering the patients' motivation for continued adherence to treatment". ${ }^{42}$ But no significant differences with symptom-triggered self-medication (also with chlordiazepoxide) was ever found by this study based on the quantity of medication consumed, time to relapse, and patient treatment satisfaction, leading its researchers to conclude that symptom-triggered self-medication (and self-monitoring) was just as safe.

Be that as it may, similar studies suggest that outpatients profit from a symptom-triggered medication therapy in terms of gaining additional motivation to enroll in counseling and medical treatments for alcoholism. ${ }^{18,19}$ There seems to be consensus within the reviewed literature that a sufficient treatment program for detoxification can reduce the severity of future AWS attacks and motivate the patients' determination to move on to long-term abstinence after completing AWS treatment. ${ }^{43,44}$

Medical complications could meanwhile lead to treatment disruptions and premature terminations. ${ }^{45}$ Benzodiazepines, presently the primary drugs of choice in AWS treatment, contain both side effects and addictive properties related to their use. ${ }^{45}$ The required daily dose of benzodiazepine is calculated based on the average daily alcohol intake. The following formula estimates the amount of a patient's alcohol consumption: ${ }^{46}$

$$
\begin{gathered}
\text { alcohol (in } \mathrm{g})=\text { volume of liquor }(\mathrm{mL}) \times 0.008 \times(\%) \\
\text { ethanol content in liquor }(\mathrm{w} / \mathrm{v})
\end{gathered}
$$

(where the percentage of alcohol in various liquors for comparative purposes is: beer [standard] $=3 \%-4 \%$; beer [strong] $=8 \%-11 \%$; wine $=5 \%-13 \%$; fortified wine $=14 \%-$ $20 \%$; spirits/hard liquor [e.g., rum, whiskey, gin, vodka, brandy $]=40 \%$; arrack $=33 \%$. Note that one standard drink contains $\sim 10 \mathrm{~g}$ of absolute alcohol or ethanol). ${ }^{47}$

On the other hand, there is growing evidence that nonbenzodiazepine GABAergic compounds represent promising medications in the treatment of alcohol-dependent patients, particularly the high-risk ones. ${ }^{48}$ The more challenging or "uncontrollable" patients are oftentimes the delirious and psychotic at Stage 3. For this reason, a typical protocol would be for the physician to halt treatment and refer the patient to the appropriate psychiatric team, which may consider the addition of an antipsychotic drug, such as haloperidol or olanzapine, in order to control agitation. Unfortunately, every antipsychotic medication bears the additional risk of lowering the seizure threshold, which is also a major concern in alcohol withdrawal. ${ }^{49}$ 
Doubtless, many more cases of disrupted or discontinued treatments have been attributed to drinking resumption, including among inpatients presenting moderate (Stage 2) to high-risk (Stage 3) symptoms. One study reported that over $20 \%$ of hospitalized AWS patients are extremely alcohol dependent. It also discovered that untreated dependence leads to repeated and longer withdrawal episodes that can further worsen alcohol abuse and dependence. ${ }^{50}$ AWS treatment alone does not produce long-term abstinence, which requires separate treatment and rehabilitation. ${ }^{51}$

One empirically understudied, but highly probable, cause of nontreatment of AWS, as well as patient drop-out, is accessibility of health care. Based on studies of untreated addictions in general, ${ }^{3,52}$ health care for AWS patients can be restricted in at least four different ways:

1. The patient's health insurance does not guarantee AWS treatment, or even alcoholism treatment for that matter. This could be because not all insurance plans are governed by the Affordable Care Act (Obamacare), which mandates free coverage for addiction treatment, while those plans that are may not be accepted by providers and treatment facilities. There are also Medicaid plans that do not cover addiction treatment at all.

2. Providers may not be accessible. AWS patients who reside far away from larger, metropolitan regions or areas might find it difficult to locate competent physicians and mental health professionals as well as suitable hospitals and other facilities. Rules govern specific treatment centers or clinicians, which also tend to restrict the number of patients they can serve.

3. Disparities exist between addiction and mental health facilities. Many addiction centers do not offer mental health treatment, while many mental health facilities do not offer addiction treatment which AWS patients will subsequently require. For most AWS patients, unless both illnesses are treated together, the likelihood of relapse is much greater.

4. Intensity and duration of AWS treatment might be limited compared to other medical conditions. Any insurance coverage might not necessarily cover AWS at the right level or for the right period of time. Patients can be discharged from treatment when insurance stops paying for it, even if the patient continues to exhibit clear symptoms or cannot yet live on his/her own.

The literature we have summarized suggests various elements and conditions that account for disrupted or prematurely terminated medical treatment of AWS patients. Clearly, both patient and provider/s are better off if treatment is timely initiated, sufficiently monitored, and concluded in accordance with the treatment plan and protocols. If so achieved, maximal health care value or benefit will accrue to the patient. The provider equally gains. This equates to the first Nash Equilibrium (with $a, w$ payoff) in Figure 2.

Without sufficient assurance that the patient will actually "cooperate" (or worse, when there is every indication that the patient cannot be trusted to do so) for whatever reason, providers risk substantial losses in terms of time, effort, and other resources, and perhaps even professional reputation. Instead, the most efficient approach under Figure 2 would be for the provider to discontinue (or opt not to initiate) treatment, as this minimizes the risk of ending up with the worst payoff when the health care benefit to the patient is practically devalued $(c, y)$. At this instance, the physician will likely refer the patient elsewhere. It is in this context that the second Nash Equilibrium in Figure 2 obtains $(d, z)$. In the case of chronic, returning drinkers, health care will likely be continued or taken over by an addiction specialist and inpatient treatment program, such as a 12-point program (e.g., Alcoholic Anonymous) ${ }^{41}$ In the case of uncontrollable patients, other medical specialists (e.g., psychiatrists) will likely be called in either to assist the health care team or fully take over the patient's treatment and medication (e.g., for psychosis or delirium). ${ }^{49}$

\section{Discussion}

In this study we modeled medical treatment of AWS as a dynamic, transactional relationship between patient and health care provider/s. We find the Stag Hunt a useful analytical model of cooperation in health care provision based on utility maximization and interactive decision-making among the key players in AWS treatments. It can be used to establish empirically verifiable predictions about the factors and conditions that promote value and quality of care as well as health-affecting behaviors during addiction cessation.

Left untreated or undertreated, many patients will experience increasing signs and symptoms of alcohol withdrawal that will vary in intensity and duration based on many factors, including severity of alcohol abuse and dependence. Although medical treatment is imperative at any withdrawal stage, and treatment strategies and plans are individualized in most cases, its completion and success depend on cooperation between patient and provider. That is partly because each party has information that the other needs, but it may not be easily or immediately accessible. The physician or provider team gathers physical-medical information from the patient, offers a diagnosis, formulates a treatment plan (and 
sometimes alternative/s), and discusses them with the patient. In doing so, the provider will rely on available information as well and his/her own appreciation of that information. The patient, on the other hand, can choose what information to disclose to the provider (e.g., drinking habits, family and peer influences, AWS symptoms and any previous episodes, existing health complications and medications, and so on). The patient will typically ask questions or make suggestions and requests that might influence the physician's perception of both the problem and the efficacy of the treatment. Above all, the patient can choose how to respond (or behave) relative to the physician's advice or prescribed treatment, with or without the latter's knowledge. While empirical research offers little evidence that decision-making in formulating treatment strategies and plans for AWS, or any other form of addiction, is usually shared between patient and doctor, ${ }^{53}$ the outcomes of these interactions will depend on the decisions and actions of each party that anticipate how the other would act and react. AWS treatment in this sense can be depicted as a game of cooperation.

For this reason, we reviewed the scant literature on AWS treatments, with attention to treatments that were disrupted or eventually discontinued. Although some variance exists in the causes of unsuccessful treatment, it is evident that the patient's choice of whether or not to comply with the doctor's advice and prescribed treatment (or the ability to do so) and the doctor's choice of how to address problems and issues affecting treatment efficacy and continuity depend on their respective cost-calculus. Built into each one's cost-calculus are the expectations of how the other party might behave or respond and the value that each party attaches to medical treatment of AWS.

The Stag Hunt model suggests two equally rational outcomes when players take risks in decision-making amid some degree of uncertainty relative to what course of action the other player might elect among a given set of alternatives and their consequences. The literature we cited appears to validate the basic premises of that model. We highlight with asterisks in Figure 3 the dual Nash Equilibria in medical treatment of AWS.

The first Nash Equilibrium $(a, w)$ is payoff-dominant because it maximizes player rewards. It is achieved when the doctor knows that the patient can (and will) fully comply with the prescribed treatment. Because of continuous monitoring and visits to the doctor, the patient becomes accountable to the doctor for his/her health-affecting behaviors. Conversely, the doctor gains sufficient familiarity with the patient's needs and condition over time and will be highly committed to the treatment when the doctor knows that a patient will return to $\mathrm{him} / \mathrm{her}$ for an indefinite period of time. Under the best-case scenario, treatment is carried out to completion and successfully evaluated in accordance with regulations and protocols. Mutual trust and assurance are essential prerequisites of cooperative interaction, which suggest that both doctor and patient will have to take some risks and assume good faith on the part of each other as treatment proceeds.

The other Nash Equilibrium $(d, z)$ might be considered a second-best outcome. It results from each player opting to discontinue treatment. It is equally rational, albeit risk dominant. Under this scenario, the patient (voluntarily or

\begin{tabular}{|c|c|c|}
\hline Players & \multicolumn{2}{|c|}{ Patient } \\
\hline Doctor & Continue Discontinue \\
\hline Continue & $a, w^{*}$ & $b, x$ \\
\hline Discontinue & $c, y$ & $d, z^{*}$ \\
\hline
\end{tabular}

Where:
$a, w=$ maximal benefit to patient or provider
$b, y=$ practically no benefit to patient or provider
$c, x=$ moderate benefit to patient or provider
$d, z=$ minimal benefit to patient or provider

*Nash Equilibrium

Figure 3 Payoff matrix in AWS treatments.

Abbreviation: AWS, Alcohol Withdrawal Syndrome. 
involuntarily) attaches less value to treatment and more to continued drinking and associated health-affecting behaviors. These could owe to a variety of systemic, sociocultural, and structural factors, including lack of motivation, domestic issues, behavioral reinforcements such as peer pressure, medication side effects, health complications, and inadequate or lack of insurance coverage. At this point, the physician might consider the patient's inability to comply as too risky or costly (e.g., as appointments and follow-ups are consistently missed, medications are not taken, potential malpractice or other lawsuits could arise). Instead of constantly pursuing the patient and making adjustments to the treatment schedule, which impose significant transaction costs, the physician might elect to discontinue or set aside treatment in various ways. These include referring the patient to addiction specialists and treatment plans that specifically address drug abuse and dependence. For the uncontrollable patients (e.g., the psychotic, extremely delirious), mental health providers, such as psychiatrists, will likely be called in. Mutual defection thus becomes cost-efficient when a physician finds that a patient is bound to indefinitely repeat noncompliant behavior, while the patient (or his/her family) might realize that seeking medical advice is too costly unless existing psychological, health, and other barriers to compliance are initially or simultaneously addressed. That is to say that patient and provider alike might initially aim for mutual cooperation, but will inevitably default to this second-best option if they cannot find mutual assurance that the other is likely to cooperate.

Unilateral defections $(b, x ; c, y)$ in Figure 3 could arise in instances where either the patient (or his/her family) or the provider elects to prematurely terminate AWS treatment. These are ostensibly win-lose scenarios, in which the reward that accrues to one party is much less than what is obtainable from the most efficient game outcome (the first Nash Equilibrium), but the loss to one transacting party is quite heavy compared to the second-best outcome (the second Nash Equilibrium). To illustrate, a doctor may unilaterally cancel treatment after some time (e.g., when the patient's insurance stops covering it). Treatment disruption or discontinuation will adversely affect a patient suffering from AWS $(c, y)$. The gain to the doctor, on the other hand, is minimal (e.g., insurance payments for a limited treatment period, the satisfaction of seeing initial progress during that short time, and so on), as potential risks and liabilities arise on the part of the doctor.

The reverse yields a similar outcome under Figure $3(b, x)$. The worst payoff for the doctor would come from committing so much time, effort, and other resources when the patient (and/or the family) has no intention of cooperating at all, except showing up for the initial, insurance-paid visit. The costs to the doctor include performing the necessary patient examinations, gathering health-related information, contacting insurer/s, developing treatment plans, determining and prescribing medications, scheduling and holding consultations, meeting and comparing notes with other providers, and patient monitoring or follow-ups.

However, because either (unilateral defection) scenario is not Nash-efficient, it is very likely that the "defecting" party will in time change course after reconsidering his/her costcalculus and gravitate toward the best or second-best options under Figure 3. For example, without the assurance that the patient will cooperate, the doctor will eventually realize that the optimal strategy would be to defect - that is, either by not attempting to initiate treatment at all or deliberately choosing to discontinue it, since both parties will reduce the risk of ending up with the worst payoff. The patient, on the other hand, is likely to return to cooperation if, for instance, his/her AWS symptoms intensify or endure, in which case noncompliance turns out to be too costly.

There is no gainsaying that further empirical study is essential to uncover the underlying game structures that occur most commonly in AWS treatments. The dual Nash Equilibria goes so far as to suggest why trust and assurance are critical in promoting collaboration in a Stag Hunt. If each player (patient and provider/s) can be relatively confident that the other will comply with what is expected of them, then mutual cooperation is a likely outcome. Otherwise, the outcome will be one of conflict or defection. Therein lies the pivotal role of information, including its communication processes, in the functioning of health care systems and in stimulating healthaffecting behaviors under conditions of risk and uncertainty. For one, how likely each player will cooperate will depend in part on the history of their past interactions (e.g., how long the patient has been with the doctor's practice) and how well that history has been communicated to and appreciated by the players. Past interactions further allow each player to estimate the degree of cooperativeness, and hence accountability, of the other. ${ }^{54}$ The organizational or environmental context of player interactions might also be considered. For example, uncooperative behavior as an inpatient can communicate signals to providers to reconsider the efficacy of an existing treatment plan. On the other hand, uncooperative behavior could be mitigated by the sterling reputation of the provider/s and facility if properly recognized by and conveyed to the patient. Finally, infinite iterations of doctor-patient interactions can help breed cooperation and mutual accountability 
in AWS treatments and services. This is because either one can expect to repeatedly encounter (and work with) each other for an indefinite period of time in the absence of any predetermined or foreseeable final visit or check-up. Thus, whether cooperation is supported at all also depends on the probability that the players would meet again. In game theory, this is referred to as the "discount parameter" or, as one economist aptly puts it, the "shadow of the future". ${ }^{55}$

This study nonetheless suggests that the effect of cooperation is distinct from the effect of time in AWS as well as other addiction-cessation treatments. Simulation games, such as the Stag Hunt, can describe and predict the evolution of cooperation where repeated encounters between patient and provider/s breed mutual trust and assurance of willingness to work toward a well-defined health promotion objective. The element of time is distinct, and equally important. Demonstrable proof of action for accountability to arise will require time. This is because cooperation between patient and doctor is an incremental process. Besides, time on its own is a critical element in treatment adherence. Studies suggest that longer treatment periods with continuous evaluations tend to bring more health-related benefits to the patient. Finite time frames can hamper cooperation, particularly when the patient chooses not to return (i.e., drop-out). On the other hand, if the patient and physician anticipate indefinitely interacting in the absence of any predetermined or foreseeable final visit, they will find future payoffs from mutual cooperation and accountability, which, in turn, fosters health promotion. Figure 4, which is adapted from van Veelen, ${ }^{56}$ illustrates the two symmetric Nash Equilibria (from Figure 3) along a continuum from which maximal payoffs (benefits) may be derived by patient $(w)$ and physician $(a)$ when interactions are indefinitely ongoing (i.e., rhythmic). In this sense, the patient-doctor relationship is truly a "coordination" game. Otherwise, disincentives to cooperation are likely to prevail and result in treatment disruptions and premature termination.

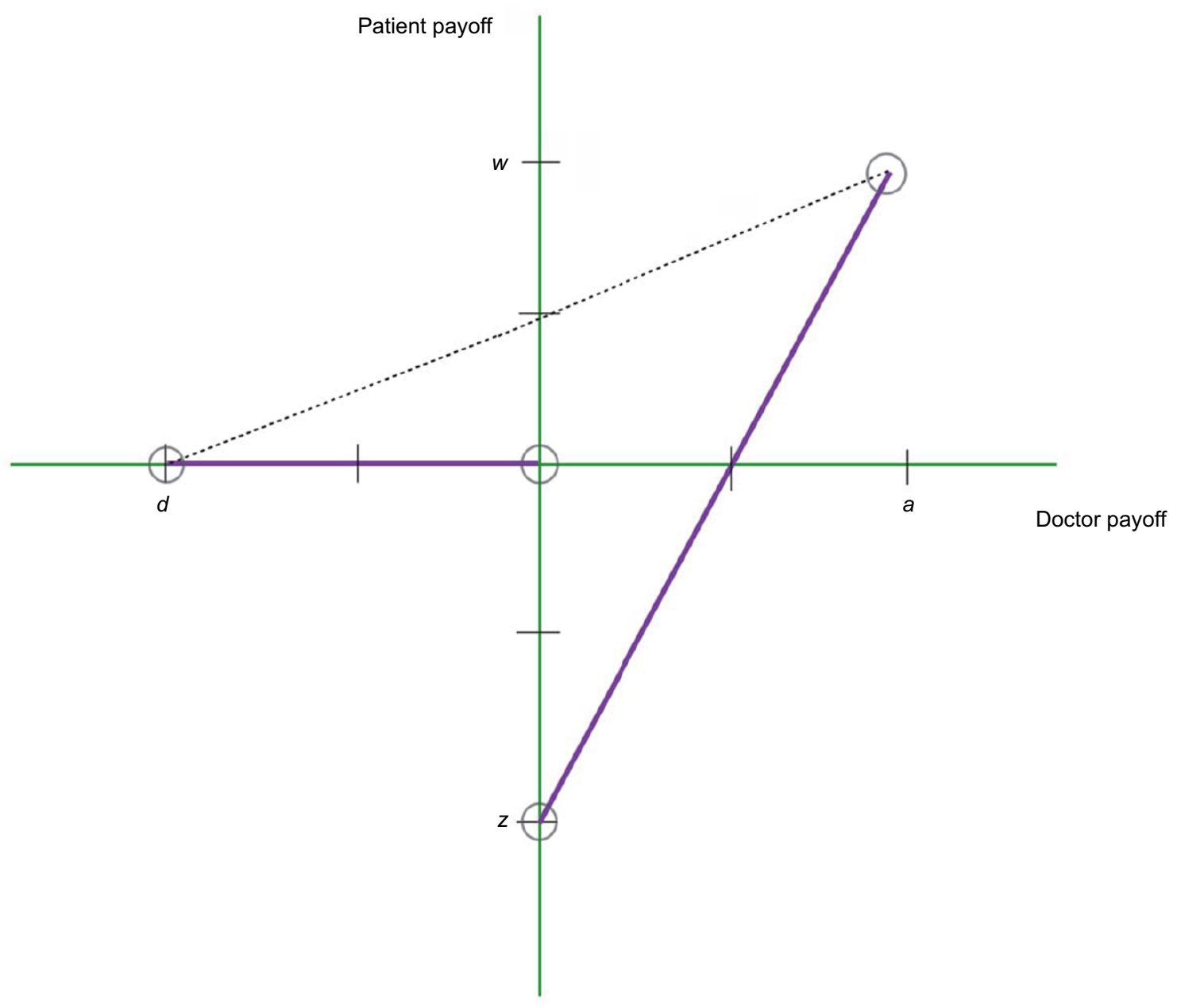

Figure 4 Payoffs in AWS treatments with indefinite time horizon.

Note: Refer to Figure 3 for corresponding notations.

Abbreviation: AWS, Alcohol Withdrawal Syndrome. 


\section{Implications for health care risk management}

We reply in the affirmative to the question raised at the outset of this study: Is treatment of AWS a Stag Hunt? We do so, particularly in instances where the patient (or health care provider) is faced with opportunities and constraints to either continue or discontinue medical treatment. The divergence and, at the same time, rationality of two Nash-efficient options highlight the key implications of the Stag Hunt for health care risk management. In discussing these implications, Figure 5 further illustrates the Stag Hunt in terms of a symmetric game with a continuous treatment strategy space, allowing players to repetitively transact with each other. In this case, patients (at various progression stages for AWS symptoms) and providers can and will separately identify their options against the opportunities and constraints before them. Each one subsequently chooses $Y$ for his/her course of action. Thus, $Y$ is a function of each player's expectation $Y e$ of the other's action.

Premises considered, the practical and theoretical insights offered by the Stag Hunt can be summed up:

1. Medical treatment of AWS is likely to be (successfully) completed, and discontinuation risks efficiently mitigated, if a patient goes to the same physician or provider team for the duration of treatment. The key factors that promote mutual cooperation in repeated plays include player anticipation of future interactions, ability to recognize each other (including each other's reputation), and recollection of past interactions.

2. Optimization of patient compliance depends in the long run on repeated contacts with a provider for an indefinite period of time (i.e., without a predetermined or foreseeable final visit or treatment). For this reason, the element of time is distinct and critical in AWS treatment.

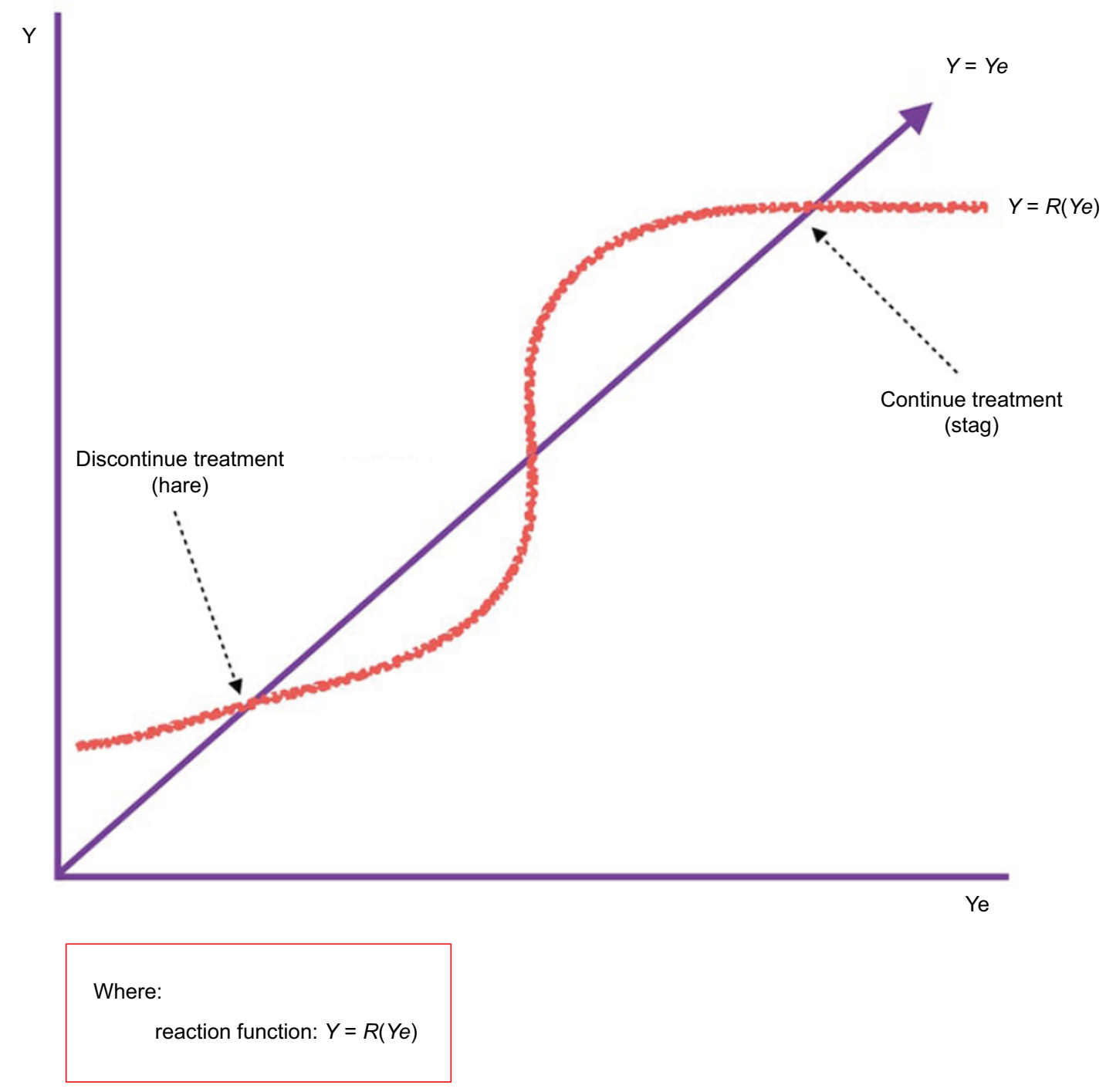

Figure 5 Stag Hunt as continuous strategy space. 
The transaction costs associated with that treatment are reduced and become manageable if the patient and provider expect to encounter each other indefinitely. Incentives will further arise for the provider to spend time and effort customizing a treatment management plan for AWS and overseeing treatment until its completion, so that future patient visits will take up less time and other resources. The patient, on the other hand, is likely to adhere to the treatment regimen if there is an expectation that the doctor will constantly monitor and evaluate his/her progress. Each will then foresee future payoffs from mutual accountability, which, in turn, fosters health promotion.

3. How the risks and uncertainties of rational and interactive decision-making are anticipated and mitigated lies at the heart of simulation games, as exemplified by the Stag Hunt. Because each player's strategy can be infinitely iterated, "strategizing" allows patient and provider to signal their commitment to cooperate (or defect) over time. Equally important, it allows for reciprocity of action based on what each one chose to pursue in their preceding interaction or encounter and, hence, the reputation they have established for themselves.

4. From a clinical approach, both addiction and mental health treatment facilities should be made available to patients suffering from AWS. Horizontal integration is crucial. Unless addiction and mental health issues are treated and managed together, the likelihood of relapse increases for the vast majority of AWS patients. This could, in turn, negatively affect their perceptions of past interactions as well as the reputation of and patient trust in the health care provider/s.

5. Continuing relationships between patient and provider are associated with a wide range of measurable treatment outcomes. On the positive side are the value and quality of care, adherence to medical treatment, reduced costs to the patient and society in general, and patient satisfaction. Negative outcomes include relapse, poorer sense of control among AWS patients, the "kindling effect", and difficulty in the application of evidence-based health care that could eventually lead to major treatment disruptions or premature terminations. Either set of outcomes might be perceived as optimal by a patient or provider depending on the balance of trust, accountability, and reciprocity in each player's cost-calculus, as well as the risk mitigation options available to them. Only if each player can be relatively assured or confident that the other will cooperate can mutual cooperation arise.
The implications of this study apply to many other addiction-cessation programs (e.g., for smoking, prohibited drugs, sex, gambling), besides alcoholism. After all, the Stag Hunt model calls attention to underlying information asymmetries, the critical role of communication, the evolution of trust and assurance, and the importance of reputation in interactive decision-making among patients and health care providers. Because the ultimate objective of simulation games is to determine the dominant solution/s for a transactional situation, the Stag Hunt illuminates why health care choices exist where neither player has anything else to gain by changing them unilaterally, and why a second-best outcome is not necessarily inferior or less efficient despite the lower payoffs. That may, in the end be, its most novel and pioneering contribution to the study and practice of health care risk management.

\section{Acknowledgments}

The author acknowledges with thanks the many helpful comments and suggestions of the three anonymous reviewers for this study as well as Nadia Etemad of Drexel University and Emily Levine of Wilmington University for proofreading the various drafts. As with any study of this nature, the usual caveat applies.

\section{Disclosure}

The author reports no conflicts of interest in this work.

\section{References}

1. Gordis E. The etiology, consequences, and treatment of alcoholism. Liver Transpl Surg. 1997;3(3):199-205.

2. Littrell J. Understanding and Treating Alcoholism, Volume I: An Empirically Based Clinician's Handbook for the Treatment of Alcoholism; Volume II: Biological, Psychological, and Social Aspects of Alcohol Consumption and Abuse. Hoboken: Taylor and Francis; 2014

3. The National Center on Addiction and Substance Abuse at Columbia University (CASA Columbia). Addiction Medicine: Closing the Gap between Science and Practice. New York, NY: CASA Columbia; 2012.

4. Giancola PR, Levinson CA, Corman MD, et al. Men and women, alcohol and aggression. Exp Clin Psychopharmacol. 2009;17(3):154-164.

5. National Research Council (NRC), Institute of Medicine (IOM), Transportation Research Board (TRB) Program Committee for a Workshop on Contributions from the Behavioral and Social Sciences in Reducing and Preventing Teen Motor Crashes. Preventing Teen Motor Crashes: Contributions from the Behavioral and Social Sciences: Workshop Report. Washington D.C.: National Academies Press; 2007.

6. Wilson IM, Graham K, Taft A. Alcohol interventions, alcohol policy and intimate partner violence: a systematic review. BMC Public Health. 2014;14:881.

7. Babor T, Caetano R, Casswell S. Alcohol: No Ordinary Commodity: Research and Public Policy. Oxford, England: Oxford University Press; 2003.

8. Organization for Economic Co-operation and Development (OECD). Health at a Glance 2017 - OECD Indicators. Available from: http:// www.keepeek.com/Digital-Asset-Management/oecd/social-issuesmigration-health/health-at-a-glance-2017_health_glance-2017-en\#. Wi7Qg2Q-czU\#page5. Accessed November 25, 2017. 
9. Hall W, Zador D. The alcohol withdrawal syndrome. Lancet. 1997;349(9069):1897-1900.

10. Becker HC. Alcohol withdrawal: neuroadaptation and sensitization. CNS Spectrums. 1999;4(1):38-65.

11. Schuckit MA. Recognition and management of withdrawal delirium (delirium tremens). N Engl J Med. 2014;371(22):2109-2113.

12. Hanwella R, de Silva V. Treatment of alcohol dependence. Ceylon Med J. 2009;54(2):63-65.

13. Hughes JR. Alcohol withdrawal seizures. Epilepsy Behav. 2009; 15(2):92-97.

14. Flora K, Stalikas A. Factors affecting substance abuse treatment across different treatment phases. Int J Psychosoc Rehabil. 2013;17(1): 89-104.

15. Newlin DB, Regalia PA, Seidman TI, Bobashev G. Control theory and addictive behavior. In: Gutkin B, Ahmed S, editors. Computational Neuroscience of Drug Addiction. New York, NY: Springer; 2012: 57-108.

16. WebMD. Alcohol and Drug Problems; 2016. Available from: http:// www.webmd.com/mental-health/addiction/tc/alcohol-and-drugproblems-topic-overview. Accessed March 01, 2017.

17. McKeon A, Frye MA, Delanty N. The alcohol withdrawal syndrome. J Neurol Neurosurg Psychiatry. 2008;79(8):854-862.

18. Holbrook AM, Crowther R, Lotter A. Meta-analysis of benzodiazepine use in the treatment of acute alcohol withdrawal. CMAJ. 1999;160(5):649-655.

19. Ntais C, Pakos E, Kyzas P, Ioannidis JP. Benzodiazepines for alcohol withdrawal. Cochrane Database Syst Rev. 2005;(3):CD005063.

20. Morgan MY, Ritson B. Alcohol and Health. London: Medical Council on Alcoholism; 1998.

21. Arfken CL, Klein C, di Menza S, Schuster CR. Gender differences in problem severity at assessement and treatment retention. J Subst Abuse Treat. 2001;20(1):53-57.

22. De Weert-Van Oene GH, Schippers GM, De Jong CA, Schrijvers GJ. Retention in substance dependence treatment: The relevance of intreatment factors. J Subst Abuse Trea. 2001;20(4):253-261.

23. Comfort M, Kaltenbach KA. Predictors of treatment outcomes for substance-abusing women: a retrospective study. Subst Abuse. 2000;21(1):33-45.

24. Fonsi Elbreder M, Carvalho de Humerez D, Laranjeira R. Analysis of the obstacles related to treatment entry, adherence and drop-out among alcoholic patients. Riv Psichiatr. 2009;44(6):351-356.

25. Driver RK. Understanding and managing alcohol withdrawal syndrome. Am Nurs Today. 2013;8:6.

26. Stark MJ. Dropping out of substance abuse treatment: a clinically oriented review. Clin Psychol Rev. 1992;12(1):93-116.

27. Mammo A, Weinbaum DF. Some factors that influence dropping out from outpatients alcoholism treatment facilities. $J$ Stud Alcohol. 1993;54(1):92-101.

28. Weisner C, Schmidt LA. Rethinking access to alcohol treatment. In: Galanter M Kaskutas LA, editors. Recent Developments in Alcoholism, Volume 15: Services Research in the Era of Managed Care. New York, NY: Kluwer Academic/Plenum Publishers; 2001:17-136.

29. Johnson P, Richter L, Herbert KD, McLellan AT, Carise DA. Telescoping of drinking-related behaviors: gender, racial/ethnic and age comparisons. Subst Use Misuse. 2005;40(8):1139-1151.

30. Pillon SC, Laranjeira RR. Formal education and nurses' attitudes towards alcohol and alcoholism in a Brazilian sample. São Paulo Medi J. 2005;123(4):175-180.

31. Hearne R, Connoly A Sheehan J. Alcohol abuse: prevalence and detection in a general hospital. $J R$ Soc Med. 2002;95(2):84-87.

32. Downey L, Rosengren DB, Donovan DM. Gender, waitlists, and outcomes for public-sector drug treatment. J Subst Abuse Treat. 2003;25(1):19-28.

33. Schneider U, Kroemer-Oldbrisch T, Wedegärtner F, Cimander KF, Wetterling T. Wishes and expectations of alcoholic patients concerning their therapy. Alcohol Alcohol. 2004;39(2):2141-2145.
34. Caprara A, Rodrigues J, Montenegro BJ. Building the relationship: Medical doctors and patients in the Family Medicine Program of Ceará, Brasil. Congress challenges of primary care oriented health systems: Innovations by educational institutions, health Professions and health Services., Londrina, Brazil;2001.

35. Blondell RD. Ambulatory detoxification of patients with alcohol dependence. Am Fam Physician. 2005;71(3):495-502.

36. Smith MS. Game Theory. In: Burgess G, Burgess H, editors. Beyond Intractability. Boulder: University of Colorado; 2003.

37. Asrafian H, Darzi A, Athanasiou T. Evidence Synthesis: Evolving Methodologies to Optimize Patient Care and Enhance Policy Decisions. In: Athanasiou T, Darzi A, editors. Evidence Synthesis in Healthcare. London: Springer-Verlag; 2001:1-47.

38. Skyrms B. The Stag Hunt and the Evolution of Social Structure. Cambridge: Cambridge University Press; 2004.

39. Baird K. Trust - At the core of the patient experience. Becker's Hospital Review. Available from: https://www.beckershospitalreview.com/ hospital-management-administration/trust-at-the-core-of-the-patientexperience.html. Accessed February 14, 2017.

40. Ridd M, Shaw A, Lewis G, Salisbury C. The patient-doctor relationship: A synthesis of the qualitative literature on patients' perspectives. $B r J$ Gen Pract. 2009;59(561):e116-e133.

41. Muncie HL Jr, Yasinian Y, Oge' L. Outpatient management of alcohol withdrawal syndrome. Am Fam Physician. 2013;88(9):589-595.

42. Elholm B, Larsen K, Hornnes N, Zierau F, Becker U. Alcohol withdrawal syndrome: symptom-triggered versus fixed-schedule treatment in an outpatient setting. Alcohol Alcohol. 2011;46(3):318-323.

43. Becker HC. Kindling in alcohol withdrawal. Alcohol Health Research World. 1998;22(1):25-33.

44. Erwin WE, Williams DB, Speir WA. Delirium tremens. Southern Med J. 1998;91(5):425-432.

45. Kattimani S, Bharadwaj B. Clinical management of alcohol withdrawal: a systematic review. Indian Psychiatry J. 2013;22(2):100-108.

46. Eyer F, Schuster T, Felgenhauer N, et al. Risk assessment of moderate to severe alcohol withdrawal - predictors for seizures and delirium tremens in the course of withdrawal. Alcohol Alcohol. 2011;46(4):427-433.

47. Bhushan K, Gangadhar BN. Acute effects of alcohol, opioid and cannabis use. In: Lal R, editor. Substance Use Disorder: Manual for Physicians. New Delhi: National Drug Depndence Treatment Center, All India Institute of Medical Sciences; 2005:1-13.

48. Leggio L, Kenna GA, Swift RM. New developments for the pharmacological treatment of alcohol withdrawal syndrome. A focus on nonbenzodiazepine GABAergic medications. Prog Neuro-psychopharmacol Biol Psychiatry. 2008;32(5):1106-1117.

49. Attard A, Torrens N, Holvey C. Alcohol Withdrawal Syndrome. London: Guy's and St. Thomas' NHS Foundation Trust; 2012.

50. Rawlani V, Vekaria S, Eisenberg S. Treatment of the hospitalized alcohol-dependent patient with alcohol withdrawal syndrome. Int $J$ In Med. 2008;8(1):1-11.

51. Finn DA, Crabbe JC. Exploring alcohol withdrawal syndrome. Alcohol Health Res World. 1997;21(2):149-156.

52. Pugh B. 4 Common barriers to addiction treatment. The Buzz. Available from: https://www.centeronaddiction.org/the-buzz-blog/4-commonbarriers-addiction-treatment. 2015. Accessed December 2, 2016.

53. Charles C, Gafni A, Whelan T. Shared decision-making in the medical encounter: what does it mean? (or it takes at least two to tango). Soc Sci Med. 1997;44(5):681-692.

54. Mendoza RL. Why any seatbelt mandate is an infinitely iterated Prisoner's Dilemma: a health economics perspective. Forum Soci Econ. 2016;45(3):1-22.

55. Dal Bo, P. Cooperation under the shadow of the future: experimental evidence from infinitely repeated games. Am Econ Rev. 2005;95(5): 1591-1604.

56. van Veelen M. Group selection, kin selection, altruism and cooperation: When inclusive fitness is right and when it can be wrong.JTheor Biol.2009; 259(3):589-600. 
Risk Management and Healthcare Policy is an international, peer-reviewed, open access journal focusing on all aspects of public health, policy, and preventative measures to promote good health and improve morbidity and mortality in the population. The journal welcomes submitted papers covering original research, basic science, clinical and epidemiological studies, reviews and evaluations, guidelines, expert opinion and commentary, case reports and extended reports. The manuscript management system is completely online and includes a very quick and fair peerreview system, which is all easy to use. Visit http://www.dovepress.com/ testimonials.php to read real quotes from published authors.

Submit your manuscript here: https://www.dovepress.com/risk-management-and-healthcare-policy-journal 